\title{
A SPLITTING THEOREM FOR ALGEBRAS OVER COMMUTATIVE VON NEUMANN REGULAR RINGS
}

\author{
WILLIAM C. BROWN
}

\begin{abstract}
Let $R$ be a commutative von Neumann ring. Let $A$ be an $R$-algebra which is finitely generated as an $R$-module and has $A / N$ separable over $R$. Here $N$ is the Jacobson radical of $A$. Then it is shown that there exists an $R$-separable subalgebra $S$ of $A$ such that $S+N=A$ and $S \cap N=0$. Further it is shown that if $T$ is another $R$-separable subalgebra of $A$ for which $T+N=A$ and $T \cap N=0$, then there exists an element $n \in N$ such that $(1-n) S(1-n)^{-1}=T$. This result is then used to determine the structure of all strong inertial coefficient rings.
\end{abstract}

Introduction. The purpose of this note is to prove the following theorem: Let $R$ be a commutative von Neumann regular ring. Let $A$ be an $R$-algebra which is finitely generated as an $R$-module and has $A / N$ separable over $R, N$ the Jacobson radical of $A$. Then there exists a separable $R$-subalgebra $S$ of $A$ such that $S+N=A$ and $S \cap N=0$. In terms of the definitions of [1], this theorem states that the pair $(R, 1)$ is a strong inertial coefficient ring if $R$ is a von Neumann regular ring. This theorem is then used to obtain a complete characterization of all strong inertial coefficient rings.

Preliminaries. Throughout this paper, any ring will be assumed to be associative and to contain an identity element. All subrings of a given ring are assumed to contain the identity of the given ring. All ring homomorphisms are assumed to take the identity to identity. $R$ will always denote a commutative ring and $A$ an $R$-algebra. We shall let $p$ and $N$ denote the Jacobson radicals of $R$ and $A$ respectively.

Let $\pi_{0}: R \rightarrow R / p$ be the natural projection of $R$ onto $R / p$. Then $R$ together with a ring homomorphism $\mathscr{E}: R / p \rightarrow R$ will be called a pair and written $(R, \mathscr{E})$ if $\pi_{0} \mathscr{E}$ is the identity map on $R / p$. The pair $(R, \mathscr{E})$ is called a strong inertial coefficient ring if for every $R$-algebra $A$ which is finitely generated as an $R$-module and has $A / N$ separable over $R$, there exists an $(R / p)$ separable subalgebra $S$ of $A$ such that $S+N=A$ and $S \cap N=0$. The basic properties of strong inertial coefficient rings can be found in [1].

Received by the editors February 8, 1972.

AMS (MOS) subject classifications (1970). Primary 13B20, 16A16, 16 A56.

Key words and phrases. von Neumann ring, strong inertial coefficient ring. 
The ring $R$ is a von Neumann regular ring if for every $z$ in $R$ there exists a $y$ in $R$ such that $z y z=z$. The proof of the main result in this paper is based on the theorems and techniques which appear in [6]. The author assumes the reader is familiar with these results.

The main result. We begin with the following lemma:

Lemma 1. Let $A$ be an $R$-algebra. Then $A$ is separable over $R$ if there exist elements $\left\{a_{1}, \cdots, a_{n}\right\}$ and $\left\{a_{1}^{\prime}, \cdots, a_{n}^{\prime}\right\}$ in $A$ such that

(1) $\sum_{i=1}^{n} a_{i}^{\prime} a_{i}=1$;

(2) for every $a \in A$, there exist constants $\hat{\lambda}_{i j}(a) \in R, i, j=1, \cdots, n$ such that

$$
a_{i} a=\sum_{j=1}^{n} \lambda_{i j} a_{j} \quad \text { and } \quad a a_{i}^{\prime}=\sum_{j=1}^{n} \lambda_{j i} a_{j}^{\prime}
$$

Proof. Let $I$ denote the kernel of the multiplication mapping $\mu$ : $A \otimes_{R} A^{\circ} \rightarrow A$. Then $A$ is separable over $R$ if and only if $0 \rightarrow I \rightarrow A \otimes_{R} A^{\circ} \rightarrow^{\mu}$ $A \rightarrow 0$ splits as $\left(A \otimes_{R} A^{\circ}\right)$-modules. That is, $A$ is separable over $R$ if and only if there exists an $\left(A \otimes_{R} A^{\circ}\right)$-module homomorphism $P: A \rightarrow A \otimes_{R} A^{\circ}$ such that $\mu P$ is the identity map on $A$. Conditions $1^{\circ}$ and $2^{\circ}$ of the lemma imply that the map $P: A \rightarrow A \otimes_{R} A^{\circ}$ defined by $P(1)=\sum_{i=1}^{n} a_{i}^{\prime} \otimes a_{i}$ is an $\left(A \otimes_{R} A^{\circ}\right.$ )-module homomorphism for which $\mu P$ is the identity map.

THEOREM 1. Let $R$ be a commutative von Neumann regular ring. Let $A$ be an $R$-algebra which is finitely generated as an $R$-module and has $A / N$ separable over $R$. Then there exists an $R$-separable subalgebra $S$ of $A$ such that $S+N=A$ and $S \cap N=0$.

Proof. Let $X(R)$ denote the decomposition space of $R[6, \mathrm{p} .8]$. Let $x \in X(R)$. If $M$ is any $R$-module, we shall let $M_{x}$ be $M \otimes_{R} R / x R$. It is well known that $\otimes_{R} R_{x}$ is an exact functor. Hence $0 \rightarrow N_{x} \rightarrow A_{x} \rightarrow(A / N)_{x} \rightarrow 0$ is an exact sequence of $R_{x}$-algebras. Since $R$ is a von Neumann regular ring, $R_{x}$ is a field. $(A / N)_{x}$ being a homomorphic image of $A / N$ is separable over $R_{x}$. Thus $N_{x}$ is the Jacobson radical of $A_{x}$. By Wedderburn's theorem, there exists an $R_{x}$-subalgebra $S_{x}$ of $A_{x}$ such that $N_{x} \oplus S_{x}=A_{x}$.

If $a \in A$, we shall denote by $a_{x}$ the image of $a$ in $A_{x}=A / x A$. Thus $a_{x}=a+\bar{x} A(\bar{x}=x R)$. Now let $\left\{a_{1}, \cdots, a_{n}\right\}$ be a set of $R$-module generators for $A$. Then if $\pi: A \rightarrow A / N$ denotes the natural projection of $A$ onto $A / N$, we have $\left\{\left(a_{1}\right)_{x}, \cdots,\left(a_{n}\right)_{x}\right\}$ generates $A_{x}$ as an $R_{x}$-module, $\left\{\pi\left(a_{1}\right)=\bar{a}_{1}\right.$, $\left.\pi\left(a_{n}\right)=\bar{a}_{n}\right\}$ generates $A / N$ as an $R$-module and $\left\{\left(\bar{a}_{1}\right)_{x}, \cdots,\left(\bar{a}_{n}\right)_{x}\right\}$ generates $(A / N)_{x}$ as an $R_{x}$-module. Since $S_{x}$ is an $R_{x}$-separable subalgebra of $A_{x}$ which is isomorphic to $(A / N)_{x}$, we can make the following statements: 
$1^{\circ}$. There exist elements $s_{1}^{x}, \cdots, s_{m(x)}^{x} \in S_{x}$ and elements $r_{i j k}^{x} \in R_{x}, i, j$, $k=1, \cdots, m(x)$ and $r_{i}^{x} \in R_{x}, i=1, \cdots, m(x)$ such that

(a) $\left\{s_{1}^{x}, \cdots, s_{m(x)}^{x}\right\}$ is a vector space basis of $S_{x}$ over $R_{x}$,

(b) $s_{i}^{x} s_{j}^{x}=\sum_{k=1}^{m(x)} r_{i j k}^{x} s_{k}^{x}$ for all $i, j=1, \cdots, m(x)$,

(c) $1_{x}=\sum_{i=1}^{m(x)} r_{i}^{x} s_{i}^{x}$.

Here $m(x)$ denotes some positive integer depending on $x$.

$2^{\circ}\left[4\right.$, Theorem 71.6]. There exist elements $r_{i j}^{x} \in R_{x}, i, j=1, \cdots, m(x)$ such that $s_{i}^{x^{\prime}}=\sum_{j=1}^{m(x)} r_{i j}^{x} s_{j}^{x}$, for $i=1, \cdots, m(x)$, satisfy the following two properties:

(a) $\sum_{j=1}^{m(x)} s_{j}^{x^{\prime}} s_{j}^{x}=1_{x}$

(b) for all $s^{x} \in S_{x}$, if $s_{i}^{x} s^{x}=\sum_{i=1}^{m(x)} \lambda_{i j}^{x} s_{j}^{x}$ for $i=1, \cdots, m(x)\left(\lambda_{i j}^{x} \in R_{x}\right)$, then $s^{x} s_{i}^{x^{\prime}}=\sum_{j=1}^{m(x)} \lambda_{j i}^{x} s_{j}^{x^{\prime}}$.

$3^{\circ}$. There exist elements $t_{i j}^{x} \in R_{x}, i=1, \cdots, n, j=1, \cdots, m(x)$, and elements $z_{i}^{x} \in N_{x}$ such that

$$
\left(a_{i}\right)_{x}-\sum_{j=1}^{m(x)} t_{i j}^{x} s_{j}^{x}=z_{i}^{x} \quad \text { for } i=1, \cdots, n .
$$

Now let $\left\{\boldsymbol{r}_{i j k}\right\},\left\{\boldsymbol{r}_{i}\right\}\left\{\boldsymbol{r}_{i j}\right\}$ and $\left\{t_{i j}\right\} \in R$ such that their images in $R_{x}$ are $\left\{r_{i j k}^{x}\right\},\left\{r_{i}^{x}\right\},\left\{r_{i j}^{x}\right\}$ and $\left\{t_{i j}^{x}\right\}$ respectively. Similarly let $\left\{s_{1}, \cdots, s_{m(x)}\right\}$ and $\left\{z_{1}, \cdots, z_{n}\right\}$ be elements in $A$ and $N$ respectively such that their images are $\left\{s_{1}^{x}, \cdots, s_{m(x)}^{x}\right\}$ and $\left\{z_{1}^{x}, \cdots, z_{n}^{x}\right\}$ in $A_{x}$. Now the elements

$$
s_{i} s_{j}-\sum_{k=1}^{m(x)} r_{i j k} s_{k}, \quad 1-\sum_{i=1}^{m(x)} r_{i} s_{i} \text { and } a_{i}-\sum_{j=1}^{m(x)} t_{i j} s_{j}-z_{i}
$$

may be viewed as global sections on the sheaf $\mathscr{A}(A)[6, \mathrm{p} .18]$ over $X(R)$. These sections are zero at $x$ and hence are zero on some open set $U$ of $X(R)$ containing $x$. Thus for all $y \in U$, the sections $s_{1}, \cdots, s_{m(x)}$ generate an $R_{y}$-subalgebra $S_{y}$ of $A_{y}$ such that $N_{y}+S_{y}=A_{y}$.

Now $s_{i}^{\prime}=\sum_{j=1}^{m(x)} r_{i j} s_{j}, i=1, \cdots, m(x)$, may also be viewed as sections on $\mathscr{A}(A)$. The section $\sum_{i=1}^{m(x)}\left(\sum_{j=1}^{m(x)} r_{i j} s_{j}\right) s_{i}-1$ is zero at $x$. Hence by shrinking $U$ if need be, we may assume for all $y \in U$,

$$
\sum_{i=1}^{m(x)}\left(s_{i}^{\prime}\right)_{y}\left(s_{i}\right)_{y}=1_{y} .
$$

By $1^{\circ}(\mathrm{b})$ and $2^{\circ}(\mathrm{b})$, we have $s_{j}^{x} s_{i}^{x^{\prime}}=\sum_{k=1}^{m(x)} r_{k j i}^{x} i_{k}^{x^{\prime}}$ for all $i, j=1, \cdots, m(x)$. Thus, by shrinking $U$ still further if need be, we can assume for all $y \in U$ and for all $i, j=1, \cdots, m(x)$,

$$
\left(s_{j}\right)_{y}\left(s_{i}^{\prime}\right)_{y}=\sum_{k=1}^{m(x)}\left(r_{k j i}\right)_{y}\left(s_{k i}^{\prime}\right)_{y}
$$


Since each $S_{y}$, for $y \in U$, is generated as an $R_{y}$-module by $\left(s_{1}\right)_{y}, \cdots$, $\left(s_{m(x)}\right)_{y}$, we get for every $a \in S_{y}$ there exist constants $\lambda_{i j}(a)$ in $R_{y}$ such that

$$
\left(s_{i}\right)_{y} a=\sum_{j=1}^{m(x)} \lambda_{i j}(a)\left(s_{j}\right)_{y} \text { and } a\left(s_{i}^{\prime}\right)_{y}=\sum_{j=1}^{m(x)} \lambda_{j i}(a)\left(s_{j}^{\prime}\right)_{y} .
$$

Thus by Lemma 1, each $S_{y}$ for $y \in U$ is a separable $R_{y}$-subalgebra of $A_{y}$. Since $R_{y}$ is a field, $S_{y}$ is semisimple. Hence $N_{y} \cap S_{y}=0$.

Since $x$ was an arbitrary point of $X(R)$, we have proven the following assertion: For each point $x$ in $X(R)$, there exists an open set $U_{x}$ in $X(R)$ containing $x$ and there exist elements $s_{1}(x), \cdots, s_{m(x)}(x), s_{1}^{\prime}(x), \cdots$, $s_{m(x)}^{\prime}(x) \in A, z_{1}(x), \cdots, z_{n}(x) \in N$, and elements $\left\{r_{i j k}(x)\right\},\left\{r_{i}(x)\right\},\left\{r_{i j}(x)\right\}$, $\left\{t_{i j}(x)\right\} \in R$ such that the elements $s_{1}(x)_{y}, \cdots, s_{m(x)}(x)_{y}$, generate an $R_{y}$ subalgebra $S_{y}$ for which $S_{y} \oplus N_{y}=A_{y}$, for all $y \in U_{x}$. Now $\left\{U_{x} \mid x \in X(R)\right\}$ is an open covering of $X(R)$. Hence by the partition property, there exist a finite number of open and closed, pairwise disjoint subsets $N_{1}, \cdots, N_{a}$ of $X(R)$ such that $\bigcup N_{i}=X(R)$ and each $N_{i}$ is contained in some $U_{x}$.

Let $x_{1}, \cdots, x_{q}$ be elements in $X(R)$ such that $N_{i} \subset U_{x_{i}}$ for $i=1, \cdots, q$. On each $N_{i}$ we may restrict the sections $s_{1}\left(x_{i}\right), \cdots, s_{m\left(x_{i}\right)}\left(x_{i}\right), s_{1}^{\prime}\left(x_{i}\right), \cdots$, $s_{m\left(x_{i}\right)}^{\prime}\left(x_{i}\right), z_{1}\left(x_{i}\right), \cdots, z_{n}\left(x_{i}\right),\left\{r_{i j k}\left(x_{i}\right)\right\}$, etc. Let $m=\max \left\{m\left(x_{1}\right), \cdots, m\left(x_{q}\right)\right\}$. Since the $N_{i}$ 's are pairwise disjoint, we may piece the sections together on each open set $N_{i}$ to form global sections

$\tilde{s}_{1}, \cdots, \tilde{s}_{m}, \tilde{s}_{1}^{\prime}, \cdots, \tilde{s}_{m}^{\prime} \in \Gamma(X(R), \mathscr{A}(A)), \quad \tilde{z}_{1}, \cdots, \tilde{z}_{n} \in \Gamma(X(R), \mathscr{A}(N))$ and $\left\{\tilde{r}_{i j k}\right\},\left\{\tilde{r}_{i}\right\},\left\{\tilde{r}_{i j}\right\},\left\{\tilde{t}_{i j}\right\} \in \Gamma(X(R), \mathscr{R}(R))$ as follows:

$$
\text { For } \alpha \in N_{i}, i=1, \cdots, q, \quad \tilde{s}_{j}(\alpha)= \begin{cases}s_{j}\left(x_{i}\right)_{\alpha} & \text { if } 1 \leqq j \leqq m\left(x_{i}\right), \\ 0 & \text { if } j>m\left(x_{i}\right) .\end{cases}
$$

The other sections are defined similarly. We now have for each $x \in X(R)$, $\left\{\tilde{s}_{1}(x), \cdots, \tilde{s}_{m}(x)\right\}$ generates an $R_{x}$-subalgebra $S_{x}$ of $A_{x}$ for which $N_{x} \oplus S_{x}=A_{x}$.

Now by [6, Theorem 4.4 and Theorem 4.5], $\Gamma(X(R), \mathscr{A}(A)) \cong A$, $\Gamma(X(R), \mathscr{A}(N)) \cong N$ and $\Gamma(X(R), \mathscr{R}(R)) \cong R$. Thus there exist elements $\hat{s}_{1}, \cdots, \hat{s}_{m} \in A, \hat{z}_{1}, \cdots, \hat{z}_{n} \in N$ and elements $\left\{\hat{r}_{i j k}\right\},\left\{\hat{r}_{i}\right\},\left\{\hat{r}_{i j}\right\},\left\{\hat{t}_{i j}\right\}$ in $R$ such that for every $x$ in $X(R)$

$$
\begin{aligned}
\tilde{s}_{i}(x) & =\left(\hat{s}_{i}\right)_{x}=\hat{s}_{i}+\bar{x} A, \\
\tilde{z}_{i}(x) & =\left(\hat{z}_{i}\right)_{x}=z_{i}+\bar{x} N, \\
\tilde{r}_{i j k}(x) & =\left(\hat{r}_{i j k}\right)_{x}=\hat{r}_{i j k}+x R, \text { etc. }
\end{aligned}
$$

Since $\bigcap_{x \in X(R)} \bar{x} A=0$, it follows easily that $S=\sum_{i=1}^{m} \hat{s}_{i} R$ is an $R$ subalgebra of $A$ such that $S+N=A$ and $S \cap N=0$. Since $S$ is isomorphic to $A / N, S$ is separable. 
COROLlaRY. Let $R$ have Jacobson radical zero. Then the pair $(R, 1)$ is a strong inertial coefficient ring if and only if $R$ is a von Neumann regular ring.

Proof. By Theorem 1 , if $R$ is a von Neumann regular ring, then $(R, 1)$ is a strong inertial coefficient ring. It follows from the proof of [3, Proposition 1] (whether $R$ is assumed Noetherian or not) that if $(R, 1)$ is a strong inertial coefficient ring, then $I=I^{2}$ for every ideal $I$ in $R$. Hence if $z \in R$, then $z R=(z R)^{2}$. So there exists a $y \in R$ such that $z y z=z$.

The Malcev analog of Theorem 1 follows immediately from [5, Corollary 2.4]. Thus under the hypotheses of Theorem 1, if $S$ and $T$ are two separable $R$-subalgebras of $A$ such that $S \oplus N=A$ and $T \oplus N=A$, then there exists an element $n \in N$ such that $(1-n) S(1-n)^{-1}=T$.

In terms of the definitions in [1], Theorems 1 and 2 may be summarized as follows: If $R$ is a von Neumann regular ring, then $(R, 1)$ is a strong inertial coefficient ring with the uniqueness property.

In [2], the author and E. Ingraham completely characterized all semilocal inertial coefficient rings. Namely, a ring $R$ is an inertial coefficient ring with finitely many maximal ideals if and only if $R$ is a finite direct sum of Hensel rings. If $(R, \mathscr{E})$ is a strong inertial coefficient ring, then $R$ is an inertial coefficient ring [1, Proposition 1]. Thus using the previous result, we get $(R, \mathscr{E})$ is a strong inertial coefficient ring with finitely many maximal ideals if and only if $R$ is a finite direct sum of split Hensel rings. In this paper, we have determined the structure of all (Jacobson) semisimple strong inertial coefficient rings. We may use these two results to give a complete characterization of strong inertial coefficient rings.

THEOREM 2. A pair $(R, \mathscr{E})$ is a strong inertial coefficient ring if and only if for every $x \in X(R), R_{x}=R / x R$ is a Hensel ring.

Proof. Suppose that for each $x$ in $X(R), R_{x}$ is a Hensel ring. Then $\left(R_{x}, \mathscr{E}_{x}\right)$ is a strong inertial coefficient ring. Thus the same proof as used in Theorem 1 with minor changes shows that $(R, \mathscr{E})$ is a strong inertial coefficient ring.

Conversely, for any pair $(R, \mathscr{E})$ we note that $X(R)=X(\mathscr{E}(R / p))$. If we assume $(R, \mathscr{E})$ is a strong inertial coefficient ring, then for any $x \in X(R)$ the pairs $\left(R_{x}, \mathscr{E}_{x}\right)$ and $(R / p, 1)$ are also strong inertial coefficient rings. By the corollary to Theorem $1, R / p$ is a von Neumann regular ring. Now

$$
0 \rightarrow p_{x} \rightarrow R_{x} \rightarrow(R / p)_{x} \rightarrow 0
$$

is exact and $(R / p)_{x}=(R / p) / x(R / p)$ is a field. Thus $R_{x}$ is a quasilocal ring. It now follows from [2, Theorem] that $R_{x}$ is a Hensel ring. 


\section{REFERENCES}

1. W. C. Brown, Strong inertial coefficient rings, Michigan Math. J. 17 (1970), 73-84. MR 41 \#8402.

2. W. C. Brown and E. C. Ingraham, A characterization of semilocal inertial coefficient rings, Proc. Amer. Math. Soc. 26 (1970), 10-14. MR 41 \#5354.

3. W. C. Brown, Some splitting theorems for algebras over commutative rings, Trans. Amer. Math. Soc. 162 (1971), 303-315.

4. C. W. Curtiss and I. Reiner, Representation theory of finite groups and associative algebras, Interscience, New York, 1962. MR 26 \#2519.

5. A. R. Magid, Pierce's representation and separable algebras, Illinois J. Math. 15 (1971), 114-121. MR 42 \#7713.

6. R. S. Pierce, Modules over commutative regular rings, Mem. Amer. Math. Soc. No. 70 (1967). MR 36 \#151.

Department of Mathematics, Michigan State University, East Lansing, MiCHIGAN 48823 